\title{
Sense Theory
}

(part 2)

Sense Function

[P-S Standard]

Egger Mielberg

egger.mielberg@gmail.com

25.07.2019

\begin{abstract}
.
The Sense Theory is not a part of traditional mathematics. It is a new paradigm of how we can formalize complex cognitive processes of the human brain. The basis of the theory is a sense function which determines sense conformity between a set of objects or/and events and a single subject (described object/event).

The sense function has a series of unique properties that can help find associative connections between trillions different-type objects/events.

By the function, we can investigate a whole process of forming a single sense of big data set of different business or scientific events.
\end{abstract}

\section{Introduction}

It has been far away in the past when a first mathematical function was formulated. Since antiquity, people used quantitative analysis in their everyday life. After hundreds of years, a great number of different mathematical sets and spaces were postulated. But none of them was not focused on such qualitative characteristic as a sense. In this article, we describe a new sense-centered function which is the main element of Sense Space (see next articles). 


\section{Problem}

In the traditional mathematical theory, a function is a relation that associates each element " $X$ " of a set " $X$ ", the domain of the function, to a single element " $y$ " of another set " $Y$ ", the codomain of the function. In many cases, set $X$, as well as set $Y$, are numeric. Such function was originally focused on how the elements of one numeric set depends on varying elements of another set.

In the advent of Al concept, many complicated and unsolvable tasks were formulated. However, neither differential calculus nor other mathematical methods helped to even approximate to a solution of the tasks. The reason for that is the codomain of the function operates elements of numeric nature whereas Al operates senses.

Below we introduce a new-paradigm function which is sense-centered.

\section{Solution}

As in classical mathematics, we need to define a "coordinate" system for operating with such an abstract object like a sense unit. Unlike rules of setting a point on the line, in Sense Theory, we need to set three conditions:

1. sense units (SU) - $\$, \$_{0}, S_{c}, S_{\ell}$

\begin{tabular}{|l|l|l|l|l|}
\hline & $\boldsymbol{S}_{\mathrm{N}}$ & $\boldsymbol{S}_{\mathrm{O}}$ & $\mathrm{S}_{\mathrm{C}}$ & $\mathrm{S}_{\not \subset}$ \\
\hline $\boldsymbol{S}_{\mathrm{N}}$ & & & & \\
\hline $\boldsymbol{S}_{\mathrm{O}(\mathrm{N})}$ & & & - & \\
\hline$S_{\mathrm{C}}$ & & & & \\
\hline$S_{\not \subset}$ & & & & \\
\hline
\end{tabular}

$$
\mathbf{S}_{\mathrm{O}(\mathrm{N})}-\mathrm{S}_{\mathrm{c}} \text { units }
$$

The table shows how many variants of the sense units can be chosen for a procedure of sense approximation. 
2. initial set (IS) is a set of Non-Sense Sets, Object Non-

Sense Sets, Complete Sense Sets and Incomplete Sense Sets which is used for first iteration of the sense approximation.

$$
I S=\left\{\boldsymbol{S}, \boldsymbol{S}_{\mathrm{O}}, \mathrm{S}_{\mathrm{C}}, \mathrm{S}_{\not \subset}\right\}_{N}
$$

3. sense direction (SD): subject or event.

The subject of the sense approximation is any element (sub-element) of Text Set which is a noun with qualitative properties.

The event of the sense approximation is a thing that happens during some time.

As well as the coordinate method of classical mathematics, in Sense Theory, there is own method, the method of sense units.

The method of sense units is the algorithm for determining the meaning (sense direction) of a text set by a chosen element or group of sense units.

For clarity's sake, we need to introduce such notation as levels of the sense approximation.

Definition 1: The first level of the sense approximation is a result of the first iteration of the calculating algorithm where the Text Set is the Initial Set.

Given:

SU - chosen variant of the sense units.

IS - chosen Text Set for the sense approximation.

SD - chosen "subject" or "event".

Definition 2: The second or $\mathrm{N}$ level of the sense approximation is a result of the second (N's) iteration of the calculating algorithm where the Text Set is the first $(\mathrm{N}-1)$ level result.

Given:

SU - chosen variant of the sense units.

IS - first ( $\mathrm{N}-1)$ level result. 
SD - chosen "subject" or "event".

Definition 3: Sense function $S_{f}$ is a sense conformity between No-Sense Set (Object No-Sense Set) and zero object $O_{0}$.

Notation: $S_{f}\left(\Phi_{(\mathbb{N})}\right)$ or $S_{f}\left(\Phi_{\text {o(N) }}\right)$ or $S_{f} \quad$, where $N=\{1,2,3, \ldots n\}$.

Like in traditional mathematics, in Sense Theory we introduce such notations as to the domain and codomain of the function. In our case, the domain of $S_{f}$ is a sense sequence. The codomain of $S_{f}$ is always one of the set of $O_{0}$. For example, in case of $S_{f}$ (frame, chassis, engine, cockpit) we have the following functional expression:

$$
\begin{gathered}
S_{f}(\text { frame, chassis, engine, cockpit })=\text { airplain }\{\} \\
\text { or } \\
S_{f}\left(\boldsymbol{S}_{(4)}\right)=\bigcirc_{\mathrm{A}}
\end{gathered}
$$

Specifying a function $\left(S_{f}\right)$ :

1. Analytical method [1]:

$$
\begin{aligned}
& S_{f}\left(\boldsymbol{S}_{\mathrm{N})}\right)=0 \\
& \text { or } \\
& S_{f}\left(\boldsymbol{S}_{\mathrm{O}(\mathrm{N})}\right)=0
\end{aligned}
$$

2. Graphical method.

This method shows the depth of the object description as well as the level of correlation with other objects with the same set of No-Sense Set elements. 


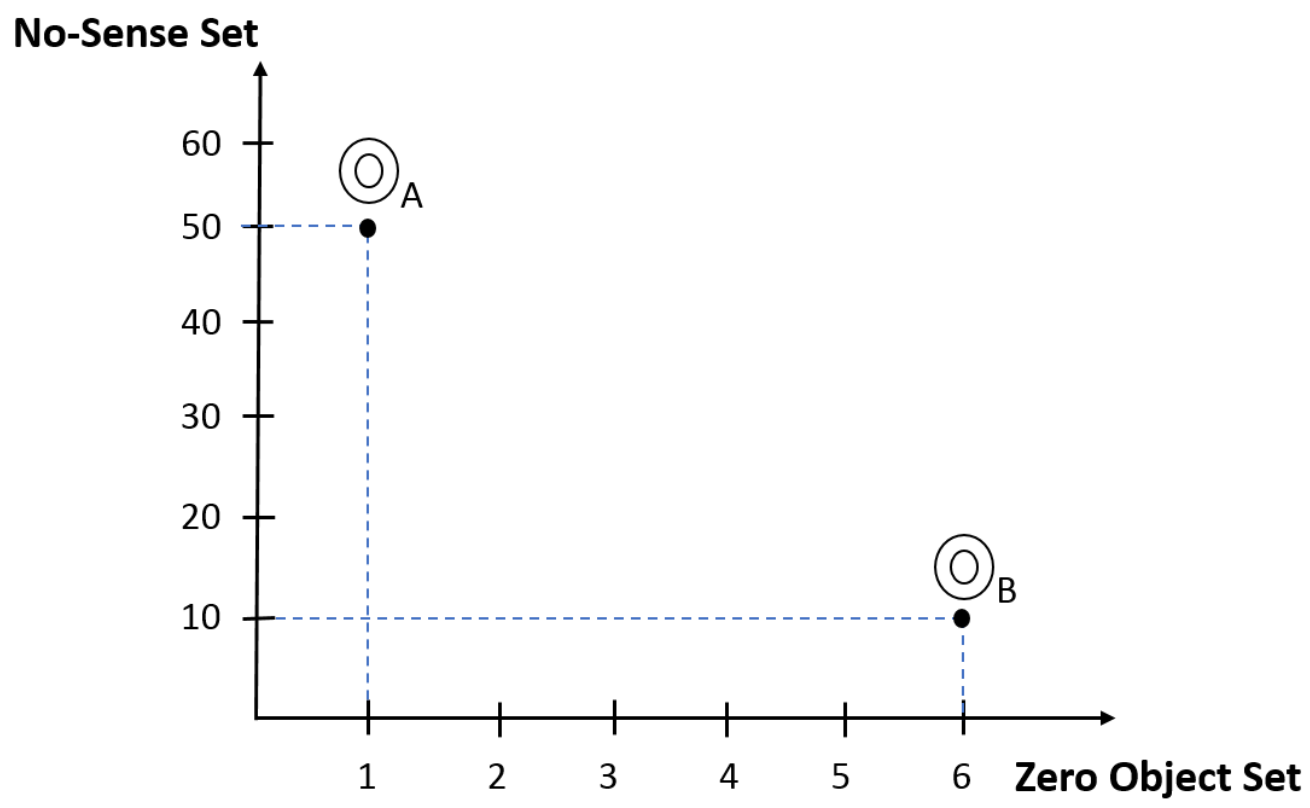

Fig.1

Definition 4: The function $S_{f}$ that has N No-Sense Set (Object No-Sense Set) elements and determines only one zero object is a $\boldsymbol{S}_{\boldsymbol{f}}$-complete function.

Definition 5: The function $S_{f}$ that has N No-Sense Set (Object No-Sense Set) elements and determines more than one zero object is a $\boldsymbol{S}_{\boldsymbol{f}}$-partial function.

It is obvious that in many practical cases the function graph will be looking like this: 


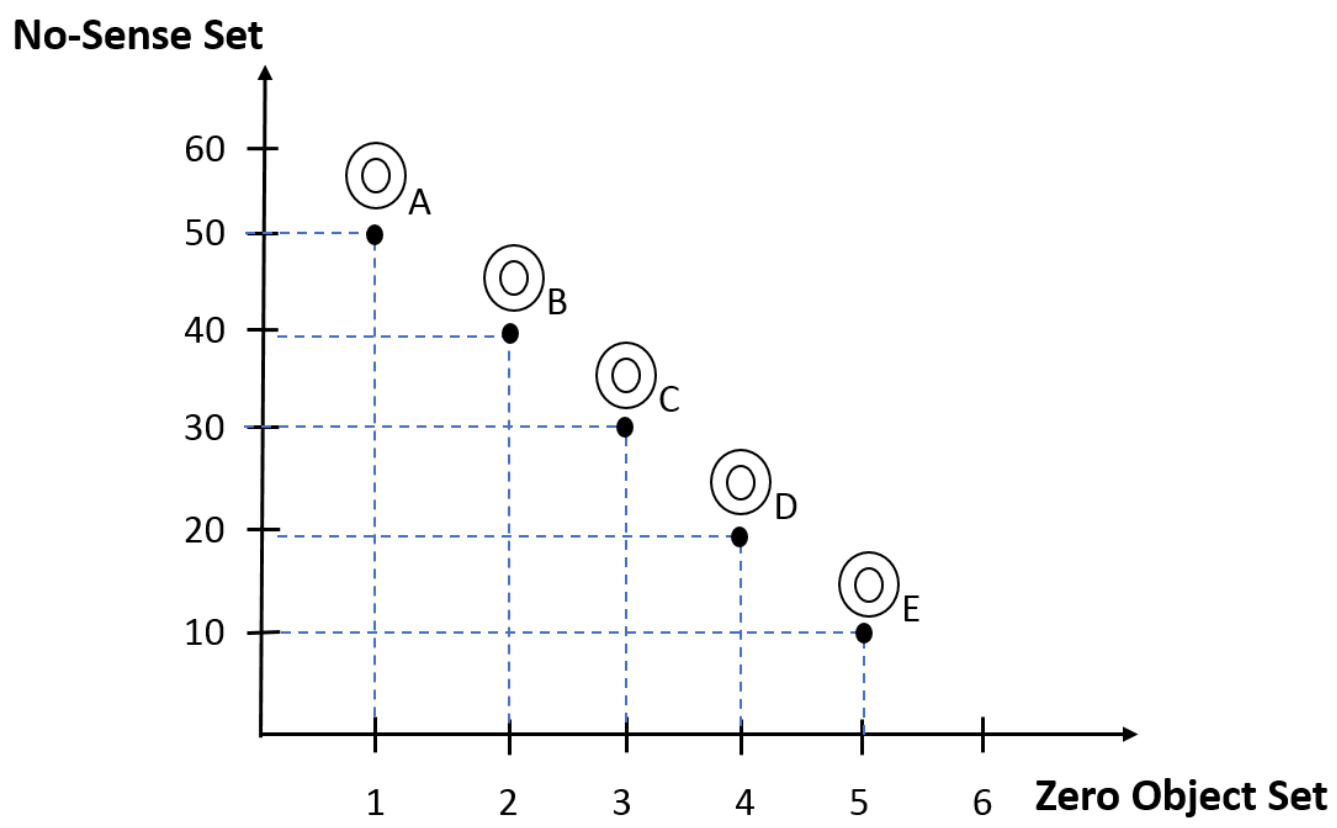

Fig.2

3. Table method.

This method allows for defining all possible combinations of No-Sense Set elements that $S_{f}$ relates to one of Object Set elements. 


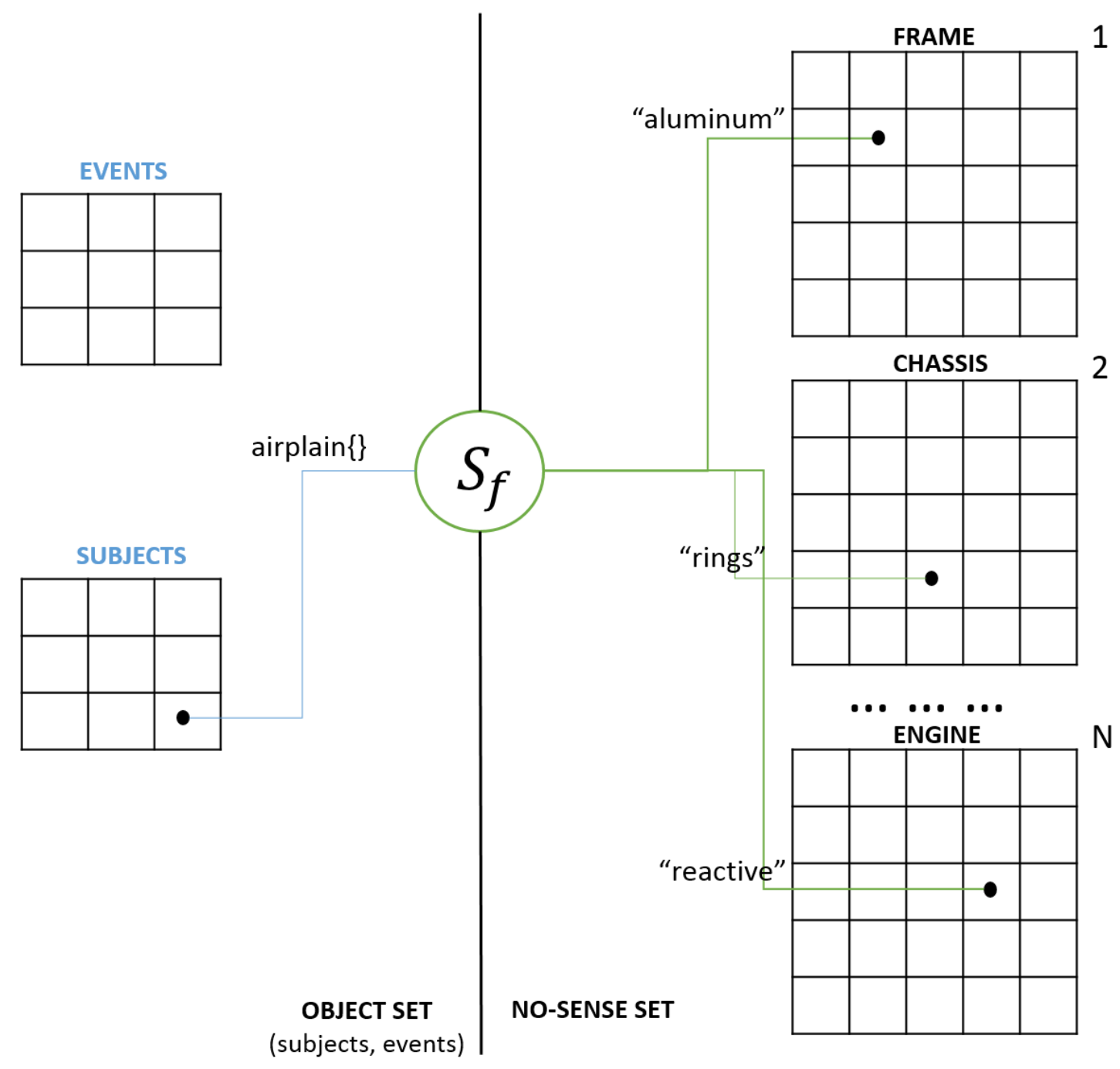

Sense determinant and limit.

Function $S_{f}^{N}$ is called sense determinant. It determines a rule of sense conformity between No-Sense Set (Object No-Sense Set) elements and a single zero object of Object Set.

Zero object in the following expression:

$$
S_{f}^{N}=\bigcirc_{\mathrm{A}}
$$

is called sense limit. It is a maximum achieved level of the sense approximation.

Unlike traditional mathematics, in Sense Theory, there is no one-to-one inverse function. Otherwise, from the following formulas:

$$
S_{f}(\bigcirc)=\$_{N}
$$




$$
\begin{gathered}
\text { or } \\
S_{f}(\bigcirc)=\$_{\mathrm{O}(\mathrm{N})}
\end{gathered}
$$

we would get the following result:

$$
\begin{gathered}
\lim _{S} \odot=\not_{N} \\
\text { or } \\
\lim _{S} \odot=\oiint_{O(N)}
\end{gathered}
$$

and

$$
\begin{gathered}
\$_{N} \odot A=S \\
\text { or } \\
S_{\text {o(N) }} \subset A=S
\end{gathered}
$$

The result is a direct contradiction to Definition 1 [1] and the main definition of the sense function.

Theorem (Equivalence of functions).

"Two functions $S_{f}^{L}$ and $S_{f}^{M}$ are equivalent if and only if the following expression is met:

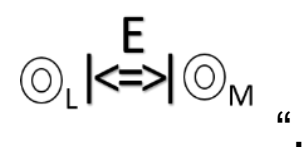

\section{Proof.}

The theorem is proofed by using two Axioms, "The Axiom of Object Limit" and "The Axiom of Object Equality".

Theorem (Nonexistence of composition functions).

"There is no composition function $S_{f}^{L}\left(S_{f}^{M}\right)$ as the following expression does not make a sense: 


$$
S_{f}(\bigcirc)=(0)
$$

\section{Proof.}

The proof of the theorem deduces from the main definition of $S_{f}$ and Definition 3 [1].

\section{Properties of $S_{f}$ :}

1. $\left.S_{f}\left(\boldsymbol{S}_{\llcorner} \cup\right) \boldsymbol{S}_{\mathrm{M}}\right)=S_{f}\left(\boldsymbol{S}_{\mathrm{M}}\left(\boldsymbol{S}_{\mathrm{L}}\right)\right.$

2. $\left.S_{f}\left(\boldsymbol{S}_{\mathrm{N}}(\bigcup) O_{N}\right)=S_{f}\left(O_{N} \bigcup\right) \boldsymbol{\$}_{\mathrm{N}}\right)$

3. $S_{f}\left(\$_{\text {o(N) }}\right)=S_{f}\left(\$_{N}\right)$ where $N=1$

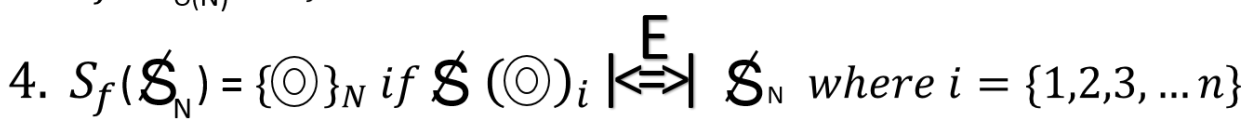

5. $S_{f}\left(\$_{N}\right) \neq \bigcirc_{N}$ if and only if $\bigcirc_{N} \subset \$_{N} \neq S$

\section{$S_{f}$ is strictly surjective.}

Theorem (Surjection of function).

"Each element of $\{\bigcirc\}_{N}$ has semantic connection to at least one sense unit of $\$_{N}\left(\$_{O(N)}\right)$

\section{Proof.}

The proof of the theorem deduces from Definition 3 and Definition 11 [1].

Definition 6: In the case when the number of $\phi_{N}\left(\phi_{\text {ovin }}\right)$ elements tends to $\mathrm{N}$, the number of semantically connected zero objects tends to 1 or 0 . In this case, $S_{f}$ is called an semantically increasing function.

$$
\lim _{\mathrm{S}} A_{N}=\{\bigcirc\}_{1} \text { or }\{\bigcirc\}_{0}
$$

Definition 7: In the case when the number of $\phi_{v}\left(\phi_{\text {own }}\right)$ elements tends to 1 , the number of semantically connected zero objects tends to $\mathrm{N}$. In this case, $S_{f}$ is called an semantically decreasing function. 


$$
\lim _{S} A_{1}=\{\bigcirc\}_{N}
$$

Definition 8: $S_{f}$ is defined if and only if there is a sense limit:

$$
\begin{gathered}
\lim _{S} \mathscr{S}_{N}=\left(\operatorname{for} S_{f}\left(\mathscr{S}_{N}\right)\right. \\
\text { or } \\
\lim _{S} \mathscr{S}_{\mathrm{O}(\mathrm{N})}=(\rho) \operatorname{for} S_{f}\left(\mathscr{S}_{\mathrm{O}(\mathrm{N})}\right)
\end{gathered}
$$

Definition 9: $S_{f}$ is not defined if and only if there is no sense limit:

$$
\begin{gathered}
\lim _{S} \mathscr{S}_{N} \neq \odot \operatorname{for} S_{f}\left(\mathscr{S}_{N}\right) \\
\text { or } \\
\lim _{S} \mathscr{S}_{O(N)} \neq \odot \operatorname{for} S_{f}\left(\mathscr{S}_{\text {o(N) }}\right)
\end{gathered}
$$

\section{Conclusion}

In this article, we presented the new "sense-focused" function. Unlike classical mathematical function, the sense-based codomain of our function is purely qualitative. It helps design different semantical models for purposes of any kind.

We hope that our decent work will help other Al researchers in their life endeavors.

\section{To be continued.}




\section{References}

[1] E. Mielberg, "Sense Theory", Part 1, 2019,

http://vixra.org/abs/1905.0105

[2] E. Titchmarsh, "The Theory of Functions", 1939,

https://archive.org/details/TheTheoryOfFunctions/page/n5

[3] S. Sternberg, "Theory of functions of a real variable", 2005,

http://www.math.harvard.edu/ shlomo/docs/Real Variables.pdf

[4] J. Harkness, F. Morley, "The Theory of Functions", 1898,

https://www.ams.org/journals/bull/1899-06-02/S0002-9904-1899-006712/S0002-9904-1899-00671-2.pdf

[5] R. Remmert, "Theory of Complex Function", 1991, https://www.matem.unam.mx/ hector/Remmert-TheoryCpxFtns.pdf

[6] I. Natanson, "Theory of Functions of a Real Variable", https://epdf.pub/theory-of-functions-of-a-realvariable345a969e7942ecf12b11e3b8055dd80725702.html

[7] C. Pinter, "A Book of Set Theory", 2014, http://matematicas.uis.edu.co/adrialba/sites/default/files/SetTheoryDover\%20Charles\%20C\%20Pinter.pdf

[8] K. Kunen, "Set Theory", 1980, https://pdfs.semanticscholar.org/8929/ab7afdb220d582e9880b098c23082d a8bc0c.pdf

[9] R. Andr'e, "Axioms and Set Theory", 2014, http://www.math.uwaterloo.ca/ randre/1aaset theory 140613.pdf

[10] H. Schwichtenberg, "Mathematical Logic", 2004, http://www.mathematik.unimuenchen.de/ schwicht/lectures/logic/ws03/ml.pdf

[11] J. Aspnes, "Notes on mathematical logic", 2010, http://www.cs.yale.edu/homes/aspnes/pinewiki/attachments/MathematicalL ogic/mathematical-logic.pdf

[12] M. Abramowitz, I. Stegun, "Handbook of Mathematical Functions with Formulas, Graphs and Mathematical Tables", 1972, http://people.math.sfu.ca/ cbm/aands/abramowitz and stegun.pdf [13] J. Nicholas, J. Hunter, J. Hargreaves, "Functions and Their Graphs", 
1997

http://sydney.edu.au/stuserv/documents/maths learning centre/functions graphs.pdf 